\title{
A Symbolic Approach to Vagueness Management
}

\author{
Mazen El-Sayed and Daniel Pacholczyk \\ University of Angers, 2 Boulevard Lavoisier, 49045 Angers Cedex 01, France \\ \{elsayed, pacho\}@univ-angers.fr
}

\begin{abstract}
We study knowledge-based systems using symbolic manyvalued logic and multiset theory. In previous papers we have proposed a symbolic representation of nuanced statements like "John is very tall". In this representation, we have interpreted some nuances of natural language as linguistic modifiers and we have defined them within a multiset context. In this paper, we continue the presentation of our symbolic model and we propose new deduction rules dealing with nuanced statements. We limit ourselves to present new generalizations of the Modus Ponens rule.
\end{abstract}

Keywords: knowledge representation and reasoning, imprecision, vagueness, many-valued logic, multiset theory.

\section{Introduction}

The development of knowledge-based systems is a rapidly expanding field in applied artificial intelligence. The knowledge base is comprised of a database and a rule base. We suppose that the database contains facts representing nuanced statements, like "Jo is very tall", to which one associates truth degrees. The nuanced statements can be represented more formally under the form " $\mathrm{x}$ is $m_{\alpha}$ A" where $m_{\alpha}$ and A are labels denoting respectively a nuance and a vague or imprecise term of natural language. The rule base contains rules of the form "if $x$ is $m_{\alpha} A$ then $y$ is $m_{\beta} B$ " to which one associates truth degrees.

Our work presents a symbolic-based model which permits a qualitative management of vagueness in knowledge-based systems. In dealing with vagueness, there are two issues of importance: (1) how to represent vague data, and (2) how to draw inference using vague data. When imprecise information is evaluated in a numerical way, fuzzy logic which is introduced by Zadeh 1112, is recognized as a good tool for dealing with aforementioned issues and performing reasoning upon common sense and vague knowledge-bases. In this logic, " $\mathrm{x}$ is $m_{\alpha} \mathrm{A}$ " is considered as a fuzzy proposition where $\mathrm{A}$ is modeled by a fuzzy set which is defined by a membership function. This one is generally defined upon a numerical scale. The nuance $m_{\alpha}$ is defined such as a fuzzy modifier [3]10]12] which represents, from the fuzzy set A, a new fuzzy set " $m_{\alpha} \mathrm{A}$ ". So, " $\mathrm{x}$ is $m_{\alpha} \mathrm{A}$ " is interpreted by Zadeh as " $\mathrm{x}$ is $\left(m_{\alpha} \mathrm{A}\right)$ " and is regarded as many-valued statement. A second formalism, refers to a symbolic many-valued logic [4|10], is used when imprecise information is evaluated in a symbolic way. This logic is the logical counterpart 
of a multiset theory introduced by De Glas [4]. In this theory, the term $m_{\alpha}$ linguistically expresses the degree to which the object $\mathrm{x}$ satisfies the term A. So, " $\mathrm{x}$ is $m_{\alpha} \mathrm{A}$ " means " $\mathrm{x}$ (is $m_{\alpha}$ ) A", and then is regarded as boolean statement. In other words, " $m_{\alpha} \mathrm{A}$ " does not represent a new vague term obtained from $\mathrm{A}$. In previous papers 78 , we have proposed a symbolic-based model to represent nuanced statements. This model is based on the many-valued logic proposed by Pacholczyk [10]. Our basic idea has been to consider that some nuances of natural language can not be interpreted as satisfaction degrees and must be instead defined such as linguistic modifiers. Firstly, we have proposed a new method to symbolically represent vague terms of natural language. The basic idea has been to associate with each vague term a new symbolic concept called "rule". This symbolic concept is an equivalent to the membership function within a fuzzy context. By using the new concept, we have defined linguistic modifiers within a multiset context. In this paper, our basic contribution has been to propose deduction rules dealing with nuanced information. For that purpose, we propose deduction rules generalizing the Modus Ponens rule in a many-valued logic proposed by Pacholczyk [10. We notice that the first version of this rule has been proposed in a fuzzy context by Zadeh [12] and has been studied later by various authors [139]. This paper is organized as follows. In Sect. 2, we present briefly the basic concepts of the M-valued predicate logic which forms the backbone of our work. Section 3 introduces briefly the symbolic representation model previously proposed. In Sect. 4 we study various types of inference rules and we propose new Generalized Modus Ponens rules in which we use only simple statements. In Sect. 5, we propose a generalized production system in which we define more Generalized Modus Ponens rules in more complex situations.

\section{M-Valued Predicate Logic}

Within a multiset context, to a vague term $\mathrm{A}$ and a nuance $m_{\alpha}$ are associated respectively a multiset $\mathbb{A}$ and a symbolic degree $\tau_{\alpha}$. So, the statement " $\mathrm{x}$ is $m_{\alpha}$ A" means that $\mathrm{x}$ belongs to multiset $\mathbb{A}$ with a degree $\tau_{\alpha}$. The M-valued predicate logic [10] is the logical counterpart of the multiset theory. In this logic, to each multiset $\mathbb{A}$ and a membership degree $\tau_{\alpha}$ are associated a M-valued predicate $\mathbf{A}$ and a truth degree $\tau_{\alpha}$-true. In this context, the following equivalence holds: $\mathrm{x}$ is $m_{\alpha} \mathrm{A} \Leftrightarrow x \in \in_{\alpha} \mathbb{A} \Leftrightarrow$ " $\mathrm{x}$ is $m_{\alpha} \mathbf{A}$ " is true $\Leftrightarrow$ " $\mathrm{x}$ is $\mathbf{A}$ " is $\tau_{\alpha}$-true. One supposes that the membership degrees are symbolic degrees which form an ordered set $\mathcal{L}_{M}=\left\{\tau_{\alpha}, \alpha \in[1, M]\right\}$. This set is provided with the relation of a total order: $\tau_{\alpha} \leq \tau_{\beta} \Leftrightarrow \alpha \leq \beta$, and whose smallest element is $\tau_{1}$ and the largest element is $\tau_{M}$. We can then define in $\mathcal{L}_{M}$ two operators $\wedge$ and $\vee$ and a decreasing involution $\sim$ as follows: $\tau_{\alpha} \vee \tau_{\beta}=\tau_{\max (\alpha, \beta)}, \tau_{\alpha} \wedge \tau_{\beta}=\tau_{\min (\alpha, \beta)}$ and $\sim \tau_{\alpha}=\tau_{M+1-\alpha}$. One obtains then a chain $\left\{\mathcal{L}_{M}, \vee, \wedge, \leq\right\}$ having the structure of De Morgan lattice [10]. On this set, an implication $\rightarrow$ and a T-norm $\mathrm{T}$ can be defined respectively as follows: $\tau_{\alpha} \rightarrow \tau_{\beta}=\tau_{\min (\beta-\alpha+M, M)}$ and $T\left(\tau_{\alpha}, \tau_{\beta}\right)=\tau_{\max (\beta+\alpha-M, 1)}$.

Example 1. For example, by choosing $\mathrm{M}=9$, we can introduce: $\mathcal{L}_{9}=\{$ not at all, little, enough, fairly, moderately, quite, almost, nearly, completely\}. 
In the following of this paper we focus our intention on the management of statements which are nuanced by linguistic modifiers. So, we consider that $m_{\alpha}$ A represents a multiset derived from $\mathrm{A}$, and " $\mathrm{x}$ is $m_{\alpha} \mathrm{A}$ " is a many-valued statement.

\section{Representation of Nuanced Statements}

Let us suppose that our knowledge base is characterized by a finite number of concepts $\mathcal{C}_{i}$. A set of terms $P_{i k} 1$ is associated with each concept $\mathcal{C}_{i}$, whose respective domain is denoted as $X_{i}$. The terms $P_{i k}$ are said to be the basic terms connected with the concept $\mathcal{C}_{i}$. As an example, basic terms such as "small", "moderate" and "tall" are associated with the particular concept "size of men". A finite set of linguistic modifiers $m_{\alpha}$ allows us to define nuanced terms, denoted as " $m_{\alpha} P_{i k}$ ". In previous papers 78 , we have proposed a symbolic-based model to represent nuanced statements of natural language. In the following, we present a short review of this model. We have proposed firstly a new method to symbolically represent vague terms. In this method, we suppose that a domain of a vague term, denoted by $\mathrm{X}$, is not necessarily a numerical scale. This domain is simulated by a "rule" (cf. Fig. 1) representing an arbitrary set of objects. Our basic idea has been to associate with each multiset $P_{i}$ a symbolic concept which represents an equivalent to the membership function in fuzzy set theory. For that, we have introduced a new concept, called "rule", which has a geometry similar to a membership L-R function and its role is to illustrate the membership graduality to the multisets. In order to define the geometry of this "rule", we use notions similar to those defined within a fuzzy context like the core, the support and the fuzzy part of a fuzzy set 12 . We define these notions within a multiset theory as follows: the core of a multiset $P_{i}$, denoted as $\operatorname{Core}\left(P_{i}\right)$, represents the elements belonging to $P_{i}$ with a $\tau_{M}$ degree, the support, denoted as $S p\left(P_{i}\right)$, contains the elements belonging to $P_{i}$ with at least $\tau_{2}$ degree, and the fuzzy part, denoted as $F\left(P_{i}\right)$, contains the elements belonging to $P_{i}$ with degrees varying from $\tau_{2}$ to $\tau_{M-1}$. We associate with each multiset a "rule" that contains the elements of its support (cf. Fig. 3). This "rule" is the union of three disjoint

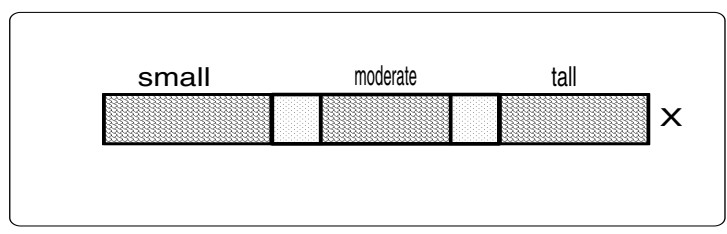

Fig. 1. Representation with "rule" of a domain X

\footnotetext{
${ }^{1}$ In the following, we use the same notation $P_{i k}$ to represent either a vague term $P_{i k}$, the multiset $\mathbb{P}_{i k}$ and the predicate $\mathbf{P}_{i k}$ associated with it.
} 


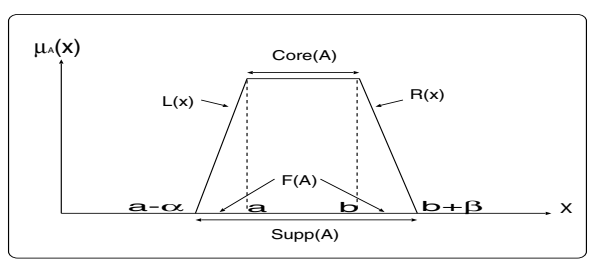

Fig. 2. A membership L-R function

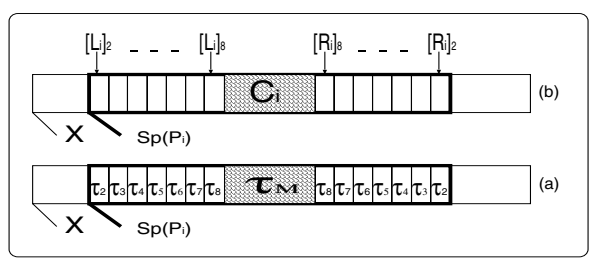

Fig. 3. Representation with "rule"

subsets: the left fuzzy part, the right fuzzy part and the core. For a multiset $P_{i}$, they are denoted respectively by $L_{i}, R_{i}$ and $C_{i}$. We suppose that the left (resp. right) fuzzy part $L_{i}$ (resp. $R_{i}$ ) is the union of M-2 subsets, denoted as $\left[L_{i}\right]_{\alpha}$ (resp. $\left[R_{i}\right]_{\alpha}$ ), which partition it. $\left[L_{i}\right]_{\alpha}$ (resp. $\left.\left[R_{i}\right]_{\alpha}\right)$ contains the elements of $L_{i}$ (resp. $R_{i}$ ) belonging to $P_{i}$ with a $\tau_{\alpha}$ degree. In order to keep a similarity with the fuzzy sets of type L-R, we choose to place, in a "rule" associated with a multiset, the subsets $\left[L_{i}\right]_{\alpha}$ and $\left[R_{i}\right]_{\alpha}$ so that the larger $\alpha$ is, the closer the $\left[L_{i}\right]_{\alpha}$ subsets and $\left[R_{i}\right]_{\alpha}$ are to the core $C_{i}$ (cf. Fig. 3). That can be interpreted as follows: the elements of the core of a term represent the typical elements of this term, and the more one object moves away from the core, the less it satisfies the term. Finally, we have denoted a multiset $P_{i}$ with which we associate a "rule" as $P_{i}=\left(L_{i}, C_{i}, R_{i}\right)$, and we have introduced symbolic parameters which enable us to describe the form of the "rule" and its position in the universe X. These parameters have a role similar to the role of numerical parameters which are used to define a fuzzy set within a fuzzy context.

\subsection{Linguistic Modifiers}

By using the "rule" concept we have defined the linguistic modifiers. We have used two types of linguistic modifiers.

- Precision Modifiers: The precision modifiers increase or decrease the precision of the basic term. We distinguish two types of precision modifiers: contraction modifiers and dilation modifiers. We use $\mathbb{M}_{6}=\left\{m_{k} \mid k \in[1 . .6]\right\}=\{$ exactly, really, $\emptyset$, more or less, approximately, vaguely $\}$ which is totally ordered by $j \leq k \Leftrightarrow m_{j} \leq m_{k}$ (Fig. 4).

- Translation Modifiers: The translation modifiers operate both a translation and precision variation (contraction or dilation) on the basic term. We use $\mathbb{T}_{9}=\left\{t_{k} \mid k \in[1 . .9]\right\}=\{$ extremely little, very very little, very little, rather little, $\emptyset$, rather, very, very very, extremely $\}$ totally ordered by $k \leq l \Leftrightarrow t_{k} \leq t_{l}$ (Fig. 5). The translation amplitudes and the precision variation amplitudes are calculated in such a way that the multisets $t_{k} P_{i}$ cover the domain $\mathrm{X}$.

In this paper, we continue to propose our model for managing nuanced statements. In the following, we focus our intention to study the problem of exploitation of nuanced statements. 


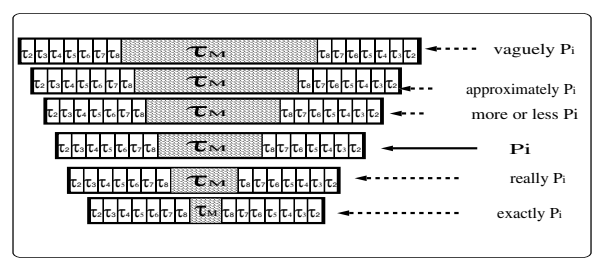

Fig. 4. Precision modifiers

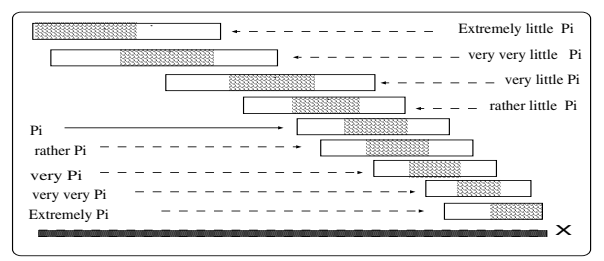

Fig. 5. Translation modifiers

\section{Exploitation of Nuanced Statements}

In this section, we treat the exploitation of nuanced information. In particular, we are interested to propose some generalizations of the Modus Ponens rule within a many-valued context [10. We notice that the classical Modus Ponens rule has the following form: If we know that $\{$ If " $x$ is $A$ " then " $y$ is $B$ " is true and " $x$ is $A$ " is true $\}$ we conclude that "y is B" is true. Within a many-valued context, a generalization of Modus Ponens rule has one of the following forms:

F1- If we know that $\left\{\right.$ If " $x$ is $A$ " then " $y$ is $B$ " is $\tau_{\beta}$-true and " $x$ is $A$ " "is $\tau_{\epsilon}$-true $\}$ and that $\left\{A^{\prime}\right.$ is more or less near to $\left.\mathrm{A}\right\}$, what can we conclude for "y is B", in other words, to what degree "y is B" is true?

F2- If we know that $\left\{\right.$ If " $x$ is $A$ " then " $y$ is $B$ " is $\tau_{\beta}$-true and " $x$ is $A$ " " is $\tau_{\epsilon}$-true $\}$ and that $\left\{A^{\prime}\right.$ is more or less near to $\left.\mathrm{A}\right\}$, can we find a $B^{\prime}$ such as $\left\{B^{\prime}\right.$ is more or less near to $\left.\mathrm{B}\right\}$ and to what degree "y is $B^{\prime} "$ is true?

These forms of Generalized Modus Ponens (GMP) rule have been studied firstly by Pacholczyk in 10. In this section, we propose new versions of GMP rule in which we use new relations of nearness.

\subsection{First GMP Rule}

In Pacholczyk's versions of GMP, the concept of nearness binding multisets A and $A^{\prime}$ is modeled by a similarity relation which is defined as follows:

Definition 1. Let $A$ and $B$ be two multisets. $A$ is said to be $\tau_{\alpha}$-similar to $B$, denoted as $A \approx_{\alpha} B$, if and only if: $\forall x \mid x \in_{\gamma} A$ and $x \in_{\beta} B \Rightarrow \min \left\{\tau_{\gamma} \rightarrow \tau_{\beta}, \tau_{\beta} \rightarrow\right.$ $\left.\tau_{\gamma}\right\} \geq \tau_{\alpha}$.

This relation generalizes the equivalence relation in a many-valued context as the similarity relation of Zadeh [12] has been in a fuzzy context. It is (1) reflexive: $A \approx_{M} A,(2)$ symmetrical: $A \approx_{\alpha} B \Leftrightarrow B \approx_{\alpha} A$, and (3) weakly transitive: $\left\{A \approx_{\alpha} B, B \approx_{\beta} C\right\} \Rightarrow A \approx_{\gamma} C$ with $\tau_{\gamma} \geq T\left(\tau_{\alpha}, \tau_{\beta}\right)$ where T is a T-norm.

By using the similarity relation to model the nearness binding between multisets, the inference rule can be interpreted as: $\{$ more the rule and the fact are true\} and $\left\{\right.$ more $A^{\prime}$ and $A$ are similar\}, more the conclusion is true. In particular, when $A^{\prime}$ is more precise than $A\left(A^{\prime} \subset A\right)$ but they are very weakly similar, any 
conclusion can be deduced or the conclusion deduced isn't as precise as one can expect. This is due to the fact that the similarity relation isn't able alone to model in a satisfactory way the nearness between $A^{\prime}$ and $A$. For that, we add to the similarity relation a new relation called nearness relation whose role is to define the nearness of $A^{\prime}$ to $A$ when $A^{\prime} \subset A$. In other words, it indicates the degree to which $A^{\prime}$ is included in $A$.

Definition 2. Let $A$ and $B$ be two multisets such that $A \subset B$. $A$ is said to be $\tau_{\alpha}$-near to $B$, denoted as $A \sqsubset_{\alpha} B$, if and only if $\left\{\forall x \in F(B), x \in_{\beta} A\right.$ and $x \in_{\gamma}$ $\left.B \Rightarrow \tau_{\alpha} \rightarrow \tau_{\beta} \leq \tau_{\gamma}\right\}$.

The nearness relation satisfies the following properties: (1) Reflexivity: $A \sqsubset_{M}$ $A$, and (2) Weak transitivity: $A \sqsubset_{\alpha} B$ and $B \sqsubset_{\beta} C \Rightarrow A \sqsubset_{\gamma} C$ with $\tau_{\gamma} \leq$ $\min \left(\tau_{\alpha}, \tau_{\beta}\right)$. In the relation $A \sqsubset_{\alpha} B$, the less the value of $\alpha$ is, the more $A$ is included in $B$. We can notice that the properties satisfied by the nearness relation are similar to those satisfied by the resemblance relation proposed by BouchonMeunier and Valverde 2] within a fuzzy context. Finally, by using similarity and nearness relations, we propose a first Generalized Modus Ponens rule.

Proposition 1. Let $A$ and $A^{\prime}$ be predicates associated with the concept $\mathcal{C}_{i}, B$ be predicate associated with the concept $\mathcal{C}_{e}$. Given the following assumptions:

1. it is $\tau_{\beta}$-true that if " $x$ is $A$ " then " $y$ is $B$ "

2. " $x$ is $A^{\prime}$ " is $\tau_{\epsilon}$-true with $A^{\prime} \approx_{\alpha} A$.

Then, we conclude: " $y$ is $B$ " is $\tau_{\delta}$-true with $\tau_{\delta}=T\left(\tau_{\beta}, T\left(\tau_{\alpha}, \tau_{\epsilon}\right)\right)$.

If the predicate $A^{\prime}$ is such that $A^{\prime} \sqsubset_{\alpha^{\prime}} A$, we conclude: " $y$ is $B$ " is $\tau_{\delta}$-true with $\tau_{\delta}=T\left(\tau_{\beta}, \tau_{\alpha^{\prime}} \longrightarrow \tau_{\epsilon}\right)$.

Example 2. Given that "really tall" $\approx_{8}$ "tall" and "really tall" $\sqsubset_{8}$ "tall", from the following rule and fact:

- if "x is tall" then "its weight is important" is true 2

- "Pascal is really tall" is quite-true,

we can deduce: "Pascal's weight is really important" is almost-true.

\subsection{GMP Rules Using Precision Modifiers}

In the previous paragraph we calculate the degree to which the conclusion of the rule is true. In the following, we present two new versions of GMP rule in which the predicate of the conclusion obtained by the deduction process is not $B$ but a new predicate $B^{\prime}$ which is more or less near to $B$. More precisely, the new predicate is derived from $\mathrm{B}$ by using precision modifier ${ }^{3}\left(B^{\prime}=m B\right)$. The first version assumes that the predicates $A$ and $A^{\prime}$ are more or less similar. In other words, $A^{\prime}$ may be less precise or more precise than $A$. The second one assumes that $A^{\prime}$ is more precise than $A$.

\footnotetext{
${ }^{2}$ In our many-valued logic, "completely true" is equivalent to "true" in classical logic.

3 The definitions of these are presented in appendix A.
} 
Proposition 2. Let $A$ and $A^{\prime}$ be predicates associated with the concept $\mathcal{C}_{i}, B$ be predicate associated with the concept $\mathcal{C}_{e}$. Let the following assumptions:

1. it is $\tau_{\beta}$-true that if " $x$ is $A$ " then " $y$ is $B$ "

2. " $x$ is $A^{\prime}$ " is $\tau_{\epsilon}$-true with $A^{\prime} \approx_{\alpha} A$.

Let $\tau_{\theta}=T\left(\tau_{\beta}, T\left(\tau_{\alpha}, \tau_{\epsilon}\right)\right)$. If $\tau_{\theta}>\tau_{1}$ then there exists a $\tau_{n(\delta)}$-dilation modifier $m$, with $\tau_{\delta} \leq T\left(\tau_{\alpha}, \tau_{\beta}\right)$, such that: " $y$ is $m B$ " is $\tau_{\epsilon^{\prime}}$-true and $\tau_{\epsilon^{\prime}}=\tau_{\delta} \longrightarrow \tau_{\theta}$.

Moreover, we have: $B \subset m B$ and $m B \approx_{\delta} B$.

This proposition prove that if we know that $A^{\prime}$ is more or less similar to $A$, without any supplementary information concerning its precision compared to $A$, the predicate of the conclusion obtained by the deduction process $(m B)$ is less precise than $B$ (i.e. $B \subset m B$ ) and which is more or less similar to $B$. In the following proposition, we assume that $A^{\prime}$ is more precise than $A$.

Proposition 3. Let $A$ and $A^{\prime}$ be predicates associated with the concept $\mathcal{C}_{i}, B$ be predicate associated with the concept $\mathcal{C}_{e}$. Let the following assumptions:

1. it is $\tau_{\beta}$-true that if " $x$ is $A$ " then " $y$ is $B$ "

2. " $x$ is $A^{\prime}$ " is $\tau_{\epsilon}$-true with $A^{\prime} \sqsubset_{\alpha} A$.

Let $\tau_{\theta}=T\left(\tau_{\beta}, \tau_{\alpha} \longrightarrow \tau_{\epsilon}\right)$. If $\tau_{\theta}>\tau_{1}$ then there exists a $\tau_{n(\delta)}$-contraction modifier $m$, with $\tau_{\delta} \geq \tau_{\beta} \longrightarrow \tau_{\alpha}$, such that: " $y$ is $m B$ " is $\tau_{\epsilon^{\prime}}$-true and $\tau_{\epsilon^{\prime}}=$ $T\left(\tau_{\delta}, \tau_{\theta}\right)$.

Moreover, we have: $m B \sqsubset_{\delta} B$.

This proposition prove that from a predicate $A^{\prime}$ which is more or less near to $A$ we obtain a predicate $m B$ which is more or less near to $B$. More precisely, if $A^{\prime}$ is more precise than $A$ then $m B$ is more precise than $B$. The previous propositions (2) and 3) present two general cases in which we consider arbitrary predicates $A^{\prime}$. In the following, we present two corollaries representing special cases of propositions 2 and 3 in which we assume that the rule is completely true and that $A^{\prime}$ is obtained from A by using precision modifiers.

Corollary 1. Let the following rule and fact:

1. it is true that if " $x$ is $A$ " then " $y$ is $B$ "

2. " $x$ is $m_{k} A$ " is $\tau_{\epsilon}$-true where $m_{k}$ is a $\tau_{\gamma_{k}}$-dilation modifier.

If $T\left(\sim \tau_{\gamma_{k}}, \tau_{\epsilon}\right)>\tau_{1}$ then we conclude:

"y is $m_{k} B$ " is $\tau_{\epsilon^{\prime}}$-true, with $\tau_{\epsilon^{\prime}}=\sim \tau_{\gamma_{k}} \longrightarrow T\left(\sim \tau_{\gamma_{k}}, \tau_{\epsilon}\right)$.

Example 3. Given the following data:

- if " $\mathrm{x}$ is tall" then "its weight is important" is true,

- "Jo is more or less tall" is moderately-true.

Then, we can deduce: "Jo's weight is more or less important" is moderately-true. 
Corollary 2. Let the following rule and fact:

1. it is true that if " $x$ is $A$ " then " $y$ is $B$ "

2. " $x$ is $m_{k} A$ " is $\tau_{\epsilon}$-true where $m_{k}$ is a $\tau_{\gamma_{k}}$-contraction modifier.

Then, we conclude that: " $y$ is $m_{k} B$ " is $\tau_{\epsilon}$-true.

Example 4. Given the following data:

- if " $\mathrm{x}$ is tall" then "its weight is important" is true,

- "Pascal is really tall" is moderately-true.

Then, we can deduce: "Pascal's weight is really important" is moderately-true.

These two corollaries present a particular form of graduality of inference. This form is known as graduality by means of linguistic modifiers [5]. It enables us to obtain, from a fact whose predicate $A^{\prime}$ is nuanced by linguistic modifiers, a conclusion whose predicate is also nuanced by linguistic modifiers.

\section{Generalized Production System}

In this section, we present some generalizations of Modus Ponens rule in more complex situations. More precisely, we study the problem of reasoning in 4 situations:

1. When the antecedent of the rule is a conjunction of statements.

2. When the antecedent is a disjunction of statements.

3. In presence of propagation of inferences. In other words, when the conclusion of the first rule is the antecedent of the second rule, and so on.

4. When a combination of imprecision is possible. In other words, when we have some rules which have the same statement in their conclusion parts.

So, we present the following 4 propositions representing inference rules in these situations.

Proposition 4 (Antecedent is a conjunction). Given the following assumptions:

1. if " $x_{1}$ is $A_{1}$ " and $\ldots$ and " $x_{n}$ is $A_{n}$ " then " $y$ is $B$ " is $\tau_{\beta}$-true,

2. for $i=1 . . n$, " $x_{i}$ is $A_{i}^{\prime}$ " is $\tau_{\epsilon_{i}}$-true,

3. for $i=1 . . n, A_{i} \approx_{\alpha_{i}} A_{i}^{\prime}$.

Then, we can deduce: " $y$ is $B$ " is $\tau_{\delta}$-true with $\tau_{\delta}=T\left(\tau_{\beta}, T\left(\tau_{\alpha_{1}}, \tau_{\epsilon_{1}}\right)\right) \wedge \ldots \wedge$ $T\left(\tau_{\beta}, T\left(\tau_{\alpha_{n}}, \tau_{\epsilon_{n}}\right)\right)$.

If, for $i=j . . k$, the predicates $A_{i}^{\prime}$ are such that $A_{i}^{\prime} \sqsubset_{\alpha_{i}^{\prime}} A_{i}$, we can deduce: " $y$ is $B$ " is $\tau_{\delta}$-true with $\tau_{\delta}=\tau_{\delta_{1}} \wedge \ldots \wedge \tau_{\delta_{n}}$ and $\tau_{\delta_{i}}=T\left(\tau_{\alpha_{i}^{\prime}} \longrightarrow \tau_{\epsilon_{i}}, \tau_{\beta}\right)$ if $i \in[j, k]$ and $\tau_{\delta_{i}}=T\left(\tau_{\beta}, T\left(\tau_{\alpha_{i}}, \tau_{\epsilon_{i}}\right)\right)$ if not. 
Proposition 5 (Antecedent is a disjunction). Given the following assumptions:

1. if " $x_{1}$ is $A_{1}$ " or $\ldots$ or " $x_{n}$ is $A_{n}$ " then " $y$ is $B$ " is $\tau_{\beta}$-true,

2. for $i=1 . . k$, " $x_{i}$ is $A_{i}^{\prime}$ " is $\tau_{\epsilon_{i}}$-true,

3. for $i=1 . . k, A_{i} \approx_{\alpha_{i}} A_{i}^{\prime}$.

Then, we can deduce: " $y$ is $B$ " is $\tau_{\delta}$-true with $\tau_{\delta}=T\left(\tau_{\beta}, T\left(\tau_{\alpha_{1}}, \tau_{\epsilon_{1}}\right)\right) \vee \ldots \vee$ $T\left(\tau_{\beta}, T\left(\tau_{\alpha_{k}}, \tau_{\epsilon_{k}}\right)\right)$.

If, for $i=j$.. L, the predicates $A_{i}^{\prime}$ are such that $A_{i}^{\prime} \sqsubset_{\alpha_{i}^{\prime}} A_{i}$, we can deduce: " $y$ is $B$ " is $\tau_{\delta}$-true with $\tau_{\delta}=\tau_{\delta_{1}} \vee \ldots \vee \tau_{\delta_{k}}$ and $\tau_{\delta_{i}}=T\left(\tau_{\alpha_{i}^{\prime}} \longrightarrow \tau_{\epsilon_{i}}, \tau_{\beta}\right)$ if $i \in[j, L]$ and $\tau_{\delta_{i}}=T\left(\tau_{\beta}, T\left(\tau_{\alpha_{i}}, \tau_{\epsilon_{i}}\right)\right)$ if not.

Proposition 6 (Propagation of inferences). Given the following assumptions:

1. if " $x$ is $A$ " then " $y$ is $B$ " is $\tau_{\beta}$-true,

2. if " $y$ is $B$ " then " $z$ is $C$ " is $\tau_{\gamma}$-true,

3. there exists $\tau_{\epsilon}>\tau_{1}$ such that " $x$ is $A$ " "is $\tau_{\epsilon}$-true,

4. there exists $\tau_{\alpha}$ such that $A \approx_{\alpha} A^{\prime}$.

Then, we can deduce: " $z$ is $C$ " is $\tau_{\delta}$-true, with $\tau_{\delta}=T\left(T\left(\tau_{\beta}, \tau_{\gamma}\right), T\left(\tau_{\alpha}, \tau_{\epsilon}\right)\right)$.

If the predicate $A^{\prime}$ is such that $A^{\prime} \sqsubset_{\alpha^{\prime}} A$, then we can deduce: " $z$ is $C$ " is $\tau_{\delta}$-true, with $\tau_{\delta}=T\left(T\left(\tau_{\beta}, \tau_{\gamma}\right), \tau_{\alpha^{\prime}} \longrightarrow \tau_{\epsilon}\right)$.

Proposition 7 (Combination of imprecisions). Given the following assumptions:

1. for $i=1 . . n$, if " $x_{i}$ is $A_{i}$ " then " $y$ is $B$ " is $\tau_{\beta_{i}}$-true,

2. for $i=1 . . n$, " $x_{i}$ is $A_{i}^{\prime}$ " is $\tau_{\epsilon_{i}}$-true,

3. for $i=1 . . n, A_{i} \approx_{\alpha_{i}} A_{i}^{\prime}$,

then we can deduce that: " $y$ is $B$ " is $\tau_{\delta}$-true with $\tau_{\delta}=T\left(\tau_{\beta_{1}}, T\left(\tau_{\alpha_{1}}, \tau_{\epsilon_{1}}\right)\right) \vee \ldots \vee$ $T\left(\tau_{\beta_{n}}, T\left(\tau_{\alpha_{n}}, \tau_{\epsilon_{n}}\right)\right)$.

If, for $i=j . . k$, the predicates $A_{i}^{\prime}$ are such that $A_{i}^{\prime} \sqsubset{ }_{\alpha_{i}^{\prime}} A_{i}$, then we can deduce: "y is $B$ " is $\tau_{\delta}$-true with $\tau_{\delta}=\tau_{\delta_{1}} \vee \ldots \vee \tau_{\delta_{n}}$ and $\tau_{\delta_{i}}=T\left(\tau_{\alpha_{i}^{\prime}} \longrightarrow \tau_{\epsilon_{i}}, \tau_{\beta_{i}}\right)$ if $i \in[j, k]$ and $\tau_{\delta_{i}}=T\left(\tau_{\beta_{i}}, T\left(\tau_{\alpha_{i}}, \tau_{\epsilon_{i}}\right)\right)$ if not.

We present below an example in which we use the GMP rules presented in this section. In this example, we use index cards written by a doctor after his consultations. From index cards $\left(I C_{i}\right)$ and some rules $\left(\mathcal{R}_{j}\right)$, we wish deduce a diagnosis.

Example 5. Let assume that we have the following rules in our base of rules.

$\mathcal{R}_{1}-$ "If the temperature is high, the patient is ill" is almost true, 
$\mathcal{R}_{2}-$ "If the tension is always high, the patient is ill" is nearly true,

$\mathcal{R}_{3}-$ "If the temperature is high and the eardrum color is very red, the disease is an otitis" is true,

$\mathcal{R}_{4}-$ "If fat eating is high, the cholesterol risk is high" is true,

$\mathcal{R}_{5}-$ "If the cholesterol risk is high, a diet with no fat is recommended" is true.

Let us assume now that we have an index card for a patient and we want to deduce a diagnosis.

$\mathcal{F}_{1}-$ "the temperature is rather high" is nearly true,

$\mathcal{F}_{2}-$ "the tension is always more or less high" is almost true,

$\mathcal{F}_{3}-$ "the eardrum color is really very red" is quite true,

$\mathcal{F}_{4}$ - "the fat eating is very very high" is moderately true.

Using the GMP rules previously presented, we deduce the following diagnosis:

$\mathcal{D}_{1}$ - "the patient is ill" is almost true,

$\mathcal{D}_{2}-$ "the disease is an otitis" is almost true,

$\mathcal{D}_{3}-$ "the cholesterol risk is high" is true,

$\mathcal{D}_{4}-$ "a diet with no fat is recommended" is true.

Let us assume that we have the following relations: "rather high" $\sqsubset_{7}$ "high", "more or less high" $\approx_{8}$ "high", "really very red" $\sqsubset_{8}$ "very red" and "very very high" $\sqsubset_{2}$ "high". Then, the diagnosis $\left(\mathcal{D}_{1}-\mathcal{D}_{4}\right)$ are obtained as follows.

- $\mathcal{D}_{1}$ is obtained by applying proposition 7 to $\left(\mathcal{F}_{1}, \mathcal{F}_{2}\right)$ and $\left(\mathcal{R}_{1}, \mathcal{R}_{2}\right)$,

- $\mathcal{D}_{2}$ is obtained by applying proposition 4 to $\left(\mathcal{F}_{1}, \mathcal{F}_{3}\right)$ and $\mathcal{R}_{3}$,

- $\mathcal{D}_{3}$ is obtained by applying proposition 1 to $\mathcal{F}_{4}$ and $\mathcal{R}_{4}$,

- $\mathcal{D}_{4}$ is obtained by applying proposition [6] to $F_{4}$ and $\left(\mathcal{R}_{4}, \mathcal{R}_{5}\right)$.

\section{Conclusion}

In this paper, we have proposed a symbolic-based model dealing with nuanced information. This model is inspired from the representation method on fuzzy logic. In previous papers, we have proposed a new representation method of nuanced statements. In this method, we have defined a vague term by symbolic parameters given by an expert in a qualitative way. By using this representation, we have defined some linguistic modifiers in a purely symbolic way. In this paper, we proposed some deduction rules dealing with nuanced statements and we presented new Generalized Modus Ponens rules. In these rules we can use either simple statements or complex statements. 


\section{References}

1. J.F. Baldwin. A new approach to approximate reasoning using fuzzy logic. Fuzzy sets and systems, 2:309-325, 1979.

2. B. Bouchon-Meunier and L. Valverde. A ressemblance approach to analogical reasoning function. Lecture notes in computer science, 1188:266-272, 1997.

3. B. Bouchon-Meunier and J. Yao. Linguistic modifiers and imprecise categories. Int. J. of intelligent systems, 7:25-36, 1992.

4. M. De-glas. Knowledge representation in fuzzy setting. Technical Report 48, LAFORIA, 1989.

5. J. Delechamp and B. Bouchon-Meunier. Graduality by means of analogical reasoning. Lecture notes in computer science, 1244:210-222, 1997.

6. D. Dubois and H. Prade. Fuzzy sets in approximate reasoning: Inference with possibility distributions. Fuzzy sets and systems, 40:143-202, 1991.

7. M. El-Sayed and D. Pacholczyk. A qualitative reasoning with nuanced information. 8th European Conference on Logics in Artificial Intelligence (JELIA 02), 283-295, Italy, 2002.

8. M. El-Sayed and D. Pacholczyk. A symbolic approach for handling nuanced information. IASTED International Conference on Artificial Intelligence and Applications (AIA 02), 285-290, Spain, 2002.

9. L.D. Lascio, A. Gisolfi, and U.C. Garcia. Linguistic hedges and the generalized modus ponens. Int. J. of intelligent systems, 14:981-993, 1999.

10. D. Pacholczyk. Contribution au traitement logico-symbolique de la connaissance. PhD thesis, University of Paris VI, 1992.

11. L.A. Zadeh. Fuzzy sets. Information and control, 8:338-353, 1965.

12. L.A. Zadeh. A theory of approximate reasoning. Int. J. Hayes, D. Michie and L. I. Mikulich (eds); Machine Intelligence, 9:149-194, 1979. 